\title{
Nirmatrelvir-ritonavir for COVID-19
}

\author{
Emily G. McDonald MD MSc, Todd C. Lee MD MPH
}

Cite as: CMAJ 2022 February 14;194:E218. doi: 10.1503/cmaj.220081; early-released February 3, 2022

1 Ritonavir-boosted nirmatrelvir (marketed as Paxlovid) is a Health Canada-approved oral antiviral medication with activity against SARS-CoV-2

Treatment is indicated for adult ( $\geq 18 \mathrm{yr}$ ) outpatients with nonhypoxic COVID-19 who are at high risk of severe disease progression (e.g., advanced age, comorbidity, unvaccinated or immunosuppressed). ${ }^{1,2}$

2 Studies recruited primarily unvaccinated participants, predated the omicron variant and have not yet undergone peer review According to available data, patients with $5 \%$ risk of hospital admission have an estimated number needed to treat to prevent 1 hospital admission of 24 (95\% confidence interval 22-29). ${ }^{3,4}$ The most common adverse effects are dysgeusia, diarrhea, vomiting, increased blood pressure and headache.

The treatment is copackaged as nirmatrelvir ( $300 \mathrm{mg}$ - two $150 \mathrm{mg}$ tablets) with ritonavir (one $100 \mathrm{mg}$ tablet); the 3 tablets are taken together twice daily for 5 days Treatment should start as soon as possible after a confirmed diagnosis of COVID-19, ideally within 5 days of symptom onset. Although observational safety data for ritonavir in pregnancy exist, no safety data exist for nirmatrelvir. In moderate renal failure (estimated glomerular filtration rate [eGFR] $30-60 \mathrm{~mL} / \mathrm{min}$ ), the dose is reduced to 1 tablet of nirmatrelvir and 1 tablet of ritonavir twice daily. Nirmatrelvir-ritonavir is contraindicated with $\mathrm{eGFR}<30 \mathrm{~mL} / \mathrm{min}$.

The ritonavir component boosts nirmatrelvir levels and is a cytochrome P450 3A4 (CYP3A4) inhibitor when taken short term, leading to important drug-drug interactions ${ }^{5}$

Particular attention should be paid to high-risk medications: antiarrhythmics (amiodarone, digoxin), oral antithrombotics (apixaban, rivaroxaban, ticagrelor), statins (atorvastatin, lovastatin, simvastatin), benzodiazepines (diazepam), opioids (methadone, fentanyl), anticonvulsants, neuropsychiatric drugs and immunosuppressants (Appendix 1, available at www.cmaj.ca/lookup/doi/10.1503/ cmaj.220081/tab-related-content).

Mitigation strategies for drug-drug interactions include dose reductions, switching or temporarily holding a drug, and therapeutic drug monitoring

Strategies should be implemented during and 3-5 days after treatment. ${ }^{5}$ Some medications (Appendix 1) reduce the efficacy of nirmatrelvirritonavir and could lead to treatment failure or virologic resistance, ${ }^{1}$ and alternative treatments for COVID-19 should be considered. ${ }^{4}$ Pharmacist consultation is recommended in many instances (Appendix 1).

\section{References}

1. The COVID-19 Treatment Guidelines Panel's statement on potential drug-drug interactions between ritonavir-boosted nirmatrelvir (Paxlovid) and concomitant medications. Bethesda (MD): National Institutes of Health; updated 2021 Dec. 30. Available: https:// www.covid19treatmentguidelines.nih.gov/therapies/statement-on -paxlovid-drug-drug-interactions/ (accessed 2022 Jan. 16).

2. Product monograph including patient medication information: Paxlovid. Kirkland (QC): Pfizer Canada ULC; 2022. Available: https:// pdf.hres.ca/dpd_pm/00064313.PDF (accessed 2022 Jan. 23).

3. Pfizer announces additional phase $2 / 3$ study results confirming robust efficacy of novel COVID-19 oral antiviral treatment candidate in reducing risk of hospitalization or death. New York: Pfizer 2021. Available: https://www.pfizer.com/news/press-release/ press-release-detail/pfizer-announces-additional-phase-23-study -results (accessed 2022 Jan. 17).

4. Lee TC, Morris AM, Grover SA, et al. Outpatient therapies for COVID-19: How do we choose? Open Forum Infect Dis 2022 Jan. 19 [Epub ahead of print]. doi: 10.1093/ofid/ofac008.

5. Fact sheet for healthcare providers: emergency use authorization for Paxlovid. Silver Spring (MD): US Food and Drug Administration; revised 2021 Dec. 22. Available: https://www.fda.gov/ media/155050/download (accessed 2022 Jan. 16).

Competing interests: Emily McDonald reports receiving salary support from the Fonds de Recherche Santé-Québec, and operating funds from the Canadian Institutes of Health Research (CIHR). Dr. McDonald also holds copyright in the software MedSafer. Todd Lee reports receiving research salary support from the Fonds de Recherche Santé-Québec, and operating funds from $\mathrm{ClHR}$ and the Interdisciplinary Initiative in Infection and Immunity (MI4). Dr. Lee also holds copyright in the software MedSafer.

This article has been peer reviewed.

Affiliations: Clinical Practice Assessment Unit (McDonald, Lee), and Divisions of Internal Medicine (McDonald) and Infectious Diseases (Lee), Department of Medicine, McGill University Health Centre, Montréal, Que.

Content licence: This is an Open Access article distributed in accordance with the terms of the Creative Commons Attribution (CC BY-NC-ND 4.0) licence, which permits use, distribution and reproduction in any medium, provided that the original publication is properly cited, the use is noncommercial (i.e., research or educational use), and no modifications or adaptations are made. See: https://creativecommons.org/licenses/by-nc-nd/4.0/

Correspondence to: Emily MCDonald, emily.mcdonald@mcgill.ca 